\title{
Information Disclosure Concerns in The Age of Wearable Computing
}

\author{
Linda Lee, JoongHwa Lee, Serge Egelman, David Wagner \\ University of California, Berkeley \\ $\{$ lnl,dlwndghk94,egelman,daw\}@ cs.berkeley.edu
}

\begin{abstract}
Wearable devices, or "wearables," bring great benefits but also potential information disclosure risks that could expose users' activities without their awareness or consent. We surveyed 1,782 Internet users about various data associated with the capabilities of popular wearable devices on the market to identify the data disclosure scenarios that users find most concerning. Our study relatively ranks potential data capture scenarios enabled by wearables and investigates the impact of the recipient of the data on the perceived risk of data disclosure. We conclude with a brief survey of users' perception of general risks associated with wearable devices, which includes factors such as safety, changes in social behaviors, and impact on fashion. To our knowledge, this is the largest user-based experiment concerning information disclosure surrounding wearables. We hope that this work will aid in the design of future user notifications, permission management, and access control schemes for wearables.
\end{abstract}

\section{INTRODUCTION}

Wearables are a growing $\$ 700$ million industry [2]. With $20 \%$ of the population owning at least one wearable and $10 \%$ using it daily [10], ubiquitous computing is becoming a reality. This trend will continue, as $52 \%$ of technology consumers are aware of wearables and $33 \%$ are likely to buy one [6].

Wearable devices enable benefits ranging from a fitnessdata inspired lifestyle to virtual-object filled augmented reality. However, wearable devices also bring new potential privacy and security risks that could expose users' activities without their awareness or consent. Although wearable devices are still in their infancy, we have already seen manifestations of these risks. Fitbit's default privacy settings inadvertently exposed information about some of their users' sexual activity [20]. Public discomfort toward facial recognition caused Google to prohibit Google Glass applications from using facial recognition [29], but still resulted in tech hate crimes against its users [37], [14].

Wearables' sensor capabilities, continuous access, and ubiquity will result in a firehose of familiar and unfamiliar types of data, at a rate which will likely dwarf the amount of data currently captured by smartphones. Bystanders in the proximity of wearable devices have already expressed interest

Permission to freely reproduce all or part of this paper for noncommercial purposes is granted provided that copies bear this notice and the full citation on the first page. Reproduction for commercial purposes is strictly prohibited without the prior written consent of the Internet Society, the first-named author (for reproduction of an entire paper only), and the author's employer if the paper was prepared within the scope of employment.

USEC '16, 21 February 2016, San Diego, CA, USA

Copyright 2016 Internet Society, ISBN 1-891562-42-8

http://dx.doi.org/10.14722/usec.2016.23006 in being notified before data about them is captured [12]. However, subjecting people to increased notifications is not a sound option, as it has shown to lead to negative effects, such as frustration and habituation [7]. An understanding of user concerns may allow for targeted and effective user communication, inform design of future permission systems, or provide insights for access control mechanisms.

The goal of this work is to motivate research on the stillmalleable future of wearable interaction models to preserve privacy and security, which we found are the top user perceived risks associated with wearable devices. Our survey of 1,782 Internet users contributes the following:

- We relatively rank 72 potential capture scenarios, which were inspired from the capabilities from the most popular wearable devices on the market at the time of the study.

- We study 4 possible data recipients to find that the recipient of the data contributes to the magnitude of overall perceived risk, but do not find statistically significant correlated factors of risk between data and recipient.

- We sketch a landscape of users' self-reported general risks regarding wearable devices and analyze responses using logistic regression models.

\section{Methodology}

We designed a survey for our IRB-approved study to capture the general public's perception of information disclosure risks associated with wearables. To determine the most concerning data disclosure scenarios, we asked participants to rate their level of concern for 6 scenarios drawn from a list of 72 possible scenarios. This was intended to elicit their perception of the severity and impact of the risk. The format of this section was based on Felt et al.'s study of user perceptions of security and privacy risks with mobile devices [15]. To get a qualitative, unbounded measurement of what people thought the most common risk associated with wearables are, we asked our participants an open-ended question.

To obtain a representative list of scenarios, 4 researchers examined the sensors, capabilities, permissions, and applications of the most popular wearable devices on the market. At the time of this study (August 2014), the most popular wearable devices included the Fitbit fitness tracker, which continuously monitors heartbeat, steps taken, and sleep patterns; the Pebble smartwatch, which can take pictures, send texts, show notifications from online, and push notifications to services; and Google Glass, which can take pictures, record 
video, and perform a subset Internet-based tasks. These devices' capabilities and requested permissions were the basis for the list of possible data capture scenarios used in this study, which we feel will be representative of what users are likely to encounter today. For a scenario to be included in this study, the scenario was required to be understandable to a user, possible to happen with today's wearable devices, and capture unique information not in other scenarios.

\section{A. Survey Questions}

We report on participants' responses to 23 questions across 4 survey sections:

- 2 reading comprehension questions

- 6 questions regarding possible wearables scenarios

- 1 open-ended wearables risk question

- 14 demographic questions

To reduce fatigue, we gave our participants a randomly selected subset of wearables scenarios. The average survey completion time was 11.5 minutes, which included four questions that we omitted from this paper due to lack of participants' familiarity with specific devices and a misguided attempt to directly compare smartphones and wearables. See the appendix for details.

Comprehension Questions Because participants might be biased to specific companies (e.g., visceral reactions to Google Glass based on popular media stories), we framed our scenarios around a fictitious wearable. The survey introduced participants to the "Cubetastic3000," which was the basis for all questions on wearables risks. We highlighted the capabilities of this device and described use cases:

Imagine that you are the proud owner of the Cubetastic3000, a new, high-tech computing device designed to be worn on your head. Imagine that you wear this device all the time, because it is very lightweight, durable, and convenient.

The Cubetastic3000 has the capability to capture video, photos, audio, and biometrics (biological data about you, such as heart rate). Just like other devices, you can install third-party applications from an app store, and these applications can use the information from the Cubetastic 3000.

With a wide range of applications, your device can do all sorts of things, such as:

- measuring heart rate, breathing, and other things to keep track of your fitness level and overall health -look at what you see to provide information about what's around you

-allow you to take notes just by telling the device what you need to remember

-take videos of you or what you see to share

- automatically take photos or video so that you can replay events that previously happened - play music that you like for you when it detects
Every once in a while, an app might do something on your Cubetastic3000 without asking you first. Depending on what the app does, your feelings could range from indifference (you don't

first. Depending on what th being very upset.
care

5. How would you feel if an app on your Cubetastic 3000 learned when, how, and how much you exercise and shared that with your work contacts, without asking you first?

Indifferent

O

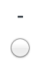

Very Upset

Fig. 1. An example of a wearable scenario question participants saw while taking the survey.

that no one is around

-infer information about you so you don't need to

log in or search for things

... and much more!

To guarantee that participants understood its capabilities, we asked two multiple-choice comprehension questions and removed participants who did not answer both correctly.

Wearable Scenarios We presented scenarios involving data captured by the Cubetastic 3000 and asked participants to rate how upset they would be if a particular type of data (e.g., how much you exercise) was shared without permission with a particular recipient (e.g., work contacts). The purpose for using this question format was to determine how upset participants would be if data were inappropriately disclosed, and the extent to which their reactions were based on the data type and recipient. Responses were reported on a 5-point Likert scale (from "indifferent" to "very upset"). Figure 1 shows an example. Specifically, questions were of the form:

"How would you feel if an app on your
Cubetastic 3000 learned (data) and shared it
with (recipient), without asking you first?"

We combined 72 data types (data) with 4 recipients (recipient) to form 288 scenarios (Table VII). Each participant answered 6 randomly drawn questions, displayed in random order. We clarified that "app" meant that the data was not shared with anyone else but a server.

Additional Questions The exit portion of the survey collected demographics (age, gender, and education) and wearable device ownership so we could control for prior exposure. We included an open-ended question about the most likely risks associated with wearable devices to capture user concerns more broadly. To avoid biasing the open-ended question, we asked it before concluding with the 10-question Internet Users' Information Privacy Concerns (IUIPC) index [27], which we used to control for participants' general privacy attitudes. However, we realize that we asked this open-ended question after exposing participants to a variety of wearables scenarios, which may have heightened their awareness of the possible risks. We talk about this more in Section 4. 


\section{B. Focus Group}

We conducted a one-hour focus group to validate our design, gauge comprehension, and measure fatigue. The focus group participants took the survey, gave feedback on the format and the content, and noted any confusion. The focus group concluded with a discussion of possible benefits and risks of wearable devices, in order to brainstorm any additional information disclosure scenarios. The Craigslistrecruited participants received $\$ 30$ in cash for their time. Of the 13 participants, 54\% were female, and ages ranged from 18 to $64(\mu=36.1, \sigma=15.3)$. Education backgrounds ranged from high school to doctorate degrees, and professions included student, artist, marketer, and court psychologist.

\section{Recruitment and Analysis Method}

We recruited 2,250 participants over August 7-13, 2014 via Amazon's Mechanical Turk. We restricted participants to those over 18, living in the United States, and having a successful HIT completion rate of $95 \%$ or above. We compensated each participant with $\$ 1.75$ upon completing the survey. Based on incorrect responses to either of the two comprehension questions, we filtered out 366 (16\% of 2,250) participants. We filtered out an additional 99 participants (4\% of 2,250) due to incomplete responses, and three participants for being under 18 , leaving us with a total sample size of 1,782 . Of these, $57.9 \%$ were male $(1,031), 41.0 \%$ were female $(731)$, and 20 participants declined to state their genders. Ages ranged from 18 to 73, with a mean of $32.1(\sigma=10.37)$. Almost half of our participants had completed a college degree or more $(49.2 \%$ of $1,782)$, which includes the $219(12.3 \%$ of 1,782$)$ who reported graduate degrees. While our sample was younger and more educated than the U.S. population as a whole, we believe it is still consistent with the U.S. Internet-using population.

In performing our analysis in the next section, we chose to focus on the very upset rate (VUR) of each scenario [15]. The VUR is defined as the percentage of participants who reported a ' 5 ' on the Likert scales. We use the VURs rather than the average of all Likert scores for the same reasons as Felt et al.: the VUR does not presume that the ratings, ranging from "indifferent" to "very upset," are linearly spaced. Additionally, most people are likely to be upset, at least a little, in all scenarios, because a device is taking action without permission (rating distribution: " $1 "=759, " 2 "=918, " 3 "=1,452$, "4" $=2,421$, " $5 "=8,344)$. Thus, the distinguishing factor is whether a participant was maximally upset. A limitation of this approach is that it only allows us to make relative comparisons between scenarios, rather than being able to definitively state how upset people might be if a single scenario were to occur. This metric is commonly used in marketing research.

\section{RESULTS}

We had at least 141 responses per data type, 2,779 per recipient, and 35 responses per each unique data type/recipient combination. In this section, we present participants' responses to the various data disclosure scenarios and discuss how various factors contributed to their risk perceptions. We conclude with self-reported general wearables concerns.
Data Type Based on our statistical models (reported later), we observed that the largest effect on participants' VURs stemmed from the data being shared, rather than with whom the data is shared. Table I lists the most and least concerning data types.

Participants were most concerned about photos and videos, especially those containing personal or embarrassing content, nudity, financial information, or information that can be used for impersonation (e.g., usernames/passwords). As seen in Table I, photos and videos accounted for five of the top ten concerns, and were almost unanimously concerning.

Participants were least concerned about data that could be collected through observations of public behavior, such as demographics (e.g., age, gender, language) or information available to advertisers (e.g., TV shows watched, music on device). As seen in Table I, participants' responses had a greater amount of variance. This greater variance and overall decreased concern may be because of uncertainty with how the data would be used, or because the financial, social, or physical consequences would be less immediate.

Although certain data is considered unanimously upsetting to have shared, it is interesting to note that no data was considered unanimously non-upsetting to have shared, nor were there any data types that evoked strong disagreement between participants (i.e., bimodal). Generally, the average concern magnitude was inversely correlated with the standard deviation, which suggests the presence of ceiling effects for the most concerning data types. For the complete ranked list of data types in this study, see Table VII.

Data Recipient A statistically significant difference in VUR exists between data shared with an application versus human recipients. On average, $42 \%$ of participants stated that they would be "very upset" if their data was shared with only an application's servers, whereas the VURs for friends $(70 \%)$, work contacts $(75 \%)$, and the public $(72 \%)$ were almost double (Table II). A chi-square test indicated that these differences were statistically significant (Table III). However, these effect sizes were small: the largest effect was between work contacts and an app's server $(\phi=0.11)$; while the VUR for sharing with work contacts was significantly higher than sharing with friends, the effect size was negligible $(\phi=0.004)$.

The statistical significance arises for two distinct reasons. Firstly, sharing data only with a server carries less social impact. For our participants, it may seem that it is shared with fewer people. Additionally, there is a class of data which may be considered odd for a human to know, but completely normal for a wearable device to know (e.g., it's okay if your Fitbit knows when you sleep, but maybe less so for your friends).

This chi-square test violates the assumption of independent observations, since participants responded to multiple scenarios with multiple recipients. But based on the randomization of treatments and large sample size, we do not believe that this significantly impacted our results. Similarly, we are unaware of a more appropriate test (beyond using mixed effects modeling), given our data format. Cochran's Q requires binary outcomes (i.e., participants would have had to answer only one question for each data recipient, preventing us from adequately controlling for data type) and a repeated measures ANOVA requires normality. Nonetheless, we repeated our analysis using one 


\begin{tabular}{|c|c|c|c|c|}
\hline Rank & Data & VUR & $\sigma$ & Distribution \\
\hline 1 & video of you unclothed & $95.97 \%$ & 0.31 & \\
\hline 2 & bank account information & $95.91 \%$ & 0.35 & \\
\hline 3 & social security number & $94.84 \%$ & 0.26 & \\
\hline 4 & video entering in a PIN at an ATM & $92.67 \%$ & 0.47 & \\
\hline 5 & photo of you unclothed & $92.59 \%$ & 0.46 & \\
\hline 6 & photo of you that is very embarrassing & $91.39 \%$ & 0.55 & \\
\hline 7 & username and password for websites & $89.55 \%$ & 0.62 & \\
\hline 8 & credit card information & $88.98 \%$ & 0.56 & \\
\hline 9 & video of you that is very embarrassing & $88.41 \%$ & 0.53 & \\
\hline 10 & photo of you at home & $87.50 \%$ & 0.60 & \\
\hline 64 & eye patterns (for eye tracking) & $40.51 \%$ & 1.27 & \\
\hline 65 & exercise patterns & $38.66 \%$ & 1.26 & \\
\hline 66 & when you are happy or having fun & $34.75 \%$ & 1.27 & \\
\hline 67 & television shows watched & $30.20 \%$ & 1.40 & \\
\hline 68 & when you are busy or interruptible & $29.50 \%$ & 1.26 & \\
\hline 69 & music on device & $28.06 \%$ & 1.43 & 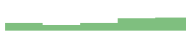 \\
\hline 70 & your heart rate & $27.50 \%$ & 1.40 & - \\
\hline 71 & age & $24.29 \%$ & 1.43 & \\
\hline 72 & language spoken & $15.86 \%$ & 1.49 & 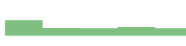 \\
\hline 73 & gender & $15.00 \%$ & 1.45 & - \\
\hline
\end{tabular}

randomly-selected data point per participant and found that our selected test was robust to this violation. Therefore, we conclude that participants were significantly more concerned about having their data seen by a human than an application, though differences between human groups such as the public, friends, and work contacts were negligible.

However, we do not claim that there are no distinctions between the friends, public, and work contact recipients. People are more comfortable sharing certain data types with certain human recipients. For instance, participants were significantly more uncomfortable sharing if they were lying, nervous, or stressed to work contacts compared to the rest of the data recipients. We believe a more fine-grained study is required to measure these effects accurately, although we have insight into the existence of these trends. Table VII shows the complete VURs and rankings of all data types by recipient.

Open-Ended Concerns To examine privacy and security concerns that may have otherwise not been captured by our scenarios, we asked participants an open-ended question:

What do you think are the most likely risks associated with wearable devices?

\begin{tabular}{|r|l|r|l|l|}
\hline Rank & Recipient & VUR & sigma & Distribution \\
\hline 1 & Work Contacts & $75.16 \%$ & 0.94 & \\
2 & Public & $72.41 \%$ & 0.98 & \\
3 & Friends & $69.47 \%$ & 1.02 & \\
4 & App's Server & $42.28 \%$ & 1.15 & \\
\hline
\end{tabular}

TABLE II. THE OVERALL UPSET RATE FOR ALL RECIPIENTS.

\begin{tabular}{|l|r|r|r|r|}
\hline Recipients & $\chi^{2}$ & p-value & $\mathrm{n}$ & $\phi$ \\
\hline Work-App & 565.910 & $<0.0001$ & 5,083 & 0.111 \\
Public-App & 481.776 & $<0.0001$ & 5,1988 & 0.093 \\
Friends-App & 381.653 & $<0.0001$ & 5,096 & 0.075 \\
Friends-Work & 20.39 & $<0.0001$ & 5,037 & 0.004 \\
Friends-Public & 5.41 & $<0.0200$ & 5,142 & 0.001 \\
Work-Public & 5.00 & $<0.0253$ & 5,129 & 0.001 \\
\hline
\end{tabular}

Participants did not receive any additional prompts and responded using a text box that did not have a character limit. Table IV shows common user concerns related to wearable 
devices. The appendix details the coding of responses. Note that participants were especially concerned with privacy and security; many answers mentioned scenarios not included in the study, but prior exposure to our questions may have heightened sensitivity to information disclosure risks:

P246: "Privacy and security of your data,
particularly for eg, [sic] stored financial/payment
or medical information"

P1256: "They can be hacked and then your security will be compromised."

Other common concerns included being unaware of what the device is collecting, doing, or which information it is using (Being Unaware), long-term health effects caused from wearing the device such as cancer from EM waves (Health), and safety hazards from wearing the device, such as distractions that cause car accidents (Safety).

P1742: Capturing and sharing data and information that you are unaware of.

P670: "Are there microwaves or some such type of waves that can pass through the brain and harm the brain? Wearing something all day can hurt that area of the body after a while."

P1038: "Becoming distracted by the devices while doing other activities that require concentration such as driving."

Interestingly, a few participants were concerned with resulting changes in social behaviors, such as dependence on devices or spending less time with loved ones (Social Impact).

P1425: I think the biggest risk is how they may effect society as a whole... a wearable technology that's always on and available may push things even further to the point where people spend less time actually interacting with loved ones, and applying their own critical thinking in certain situations, instead always relying on their devices.

The landscape of users' perceived risks associated with wearables is broad, encompassing concepts outside of privacy and security. We therefore hope this motivates other researchers to investigate these other risks.

Demographics Factors A participant's self-reported level of privacy concern - as determined by the IUIPC scale [27]is the biggest demographic predictor of VURs. A Spearman correlation yielded a statistically significant effect between average IUIPC scores and average VUR $(\rho=0.446, p<0.0005)$, which suggests responses to questions were mostly based on privacy preferences. Age was another significant predictor of VUR $(\rho=0.121, p<0.0005)$, but we suspect that this effect

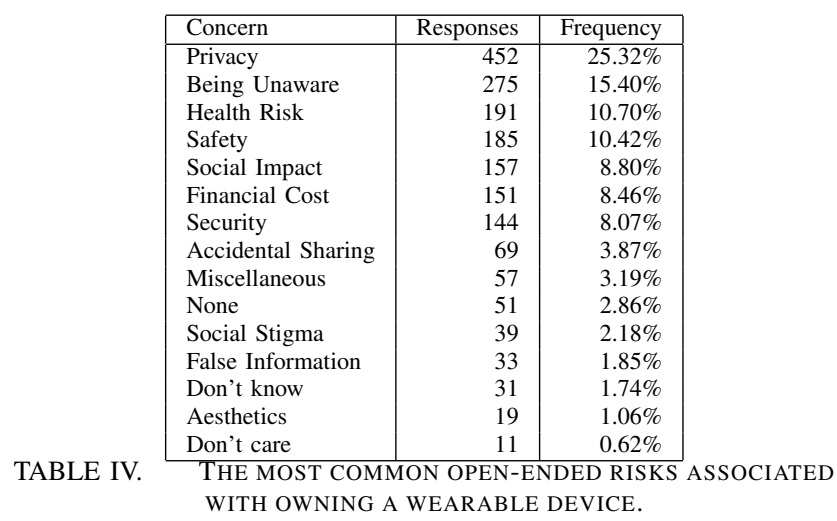

\begin{tabular}{|l|r|r|r|}
\hline Parameters & $\chi^{2}$ & $d f$ & QIC \\
\hline \hline (Intercept) & 423.96 & 1 & $13,209.1$ \\
\hline (Intercept) & 207.07 & 1 & $12,551.49$ \\
IUIPC (covariate) & 368.5 & 1 & \\
Gender (covariate) & 6.30 & 1 & \\
\hline (Intercept) & 411.66 & 1 & $12,458.86$ \\
Data Recipient & 599.72 & 3 & \\
\hline (Intercept) & 418.02 & 1 & $11,382.75$ \\
Data Type & $1,141.40$ & 71 & \\
\hline (Intercept) & 66.18 & 1 & $9,609.65$ \\
Data Recipient & 617.25 & 3 & \\
Data Type & $1,288.51$ & 71 & \\
IUIPC (covariate) & 105.73 & 1 & \\
Gender (covariate) & 9.74 & 1 & \\
IUIPC $\times$ Gender & 8.33 & 1 & \\
\hline
\end{tabular}

TABLE V. GOODNESS-OF-FIT METRICS FOR VARIOUS BINARY LOGISTIC MODELS OF OUR DATA USING GENERAL ESTIMATING EQUATIONS TO ACCOUNT FOR REPEATED MEASURES. THE COLUMNS REPRESENT THE

WALD TEST STATISTIC FOR EACH PARAMETER AND THE OVERALL

QUASI-AKAIKE INFORMATION CRITERION (QIC) FOR EACH MODEL. EACH PARAMETER LISTED WAS STATISTICALLY SIGNIFICANT AT $p<0.005$

is due to the significant correlation between age and IUIPC scores $(\rho=0.188, p<0.0005)$. Others have observed that older individuals tend to be more privacy protective [41].

While we initially observed an effect on VURs based on whether or not participants claimed to already own wearables $(57.0 \%$ vs. $60.8 \%$, respectively; Mann-Whitney $U=202,896, p<0.032)$, this difference did not remain significant upon correcting for multiple testing (Bonferroni corrected $\alpha=0.01$ ). The effect of a participant's gender also did not remain significant upon correcting for multiple testing. We observed no correlation between a participant's education level and VUR.

Regression Models To examine the relative effect of each factor on participants' VURs, we constructed several statistical models to predict whether a participant would be "very upset" with a given scenario based on the data type, data recipient, and their demographic factors (i.e., age, education, gender, and privacy attitudes). We performed binary logistic regressions using generalized estimating equations, which account for our repeated measures experimental design (i.e., each participant contributed multiple data points).

We created several models using two independent variables as predictors: data and recipient. This resulted in a total of 72 types of data shared with 4 possible recipients. Demographic factors used as covariates are: age, gender, education, wearable 
device ownership (yes/no), and mean IUIPC score. For each model, we performed Wald's test to examine the model effects attributable to each of these parameters. The covariates that had an observable effect on our models were participants' gender and IUIPC scores, which exhibited an interaction effect with each other. Thus, we opted to remove the other covariates from our analysis. Table $\mathrm{V}$ shows the various models that we examined and the Quasi-Akaike Information Criterion (QIC), which is a goodness-of-fit metric for model selection that also accounts for complexity (lower relative values indicate better fit). As shown, the type of data being shared (data type) was found to be the strongest predictor of a high VUR.

While these models illustrate the relative weights that users place on information when determining whether a scenario is truly upsetting, one shortcoming is this approach's generalizability: data types are limited to what we specifically chose for this study. To make our data set more generalizable to other use cases, we coded each data type in two ways: in terms of broad descriptions of the type of data (e.g., video, audio, etc.) and the type of risk it presents. Two researchers agreed on a codebook and independently coded each of the 72 data types. The data types fell into six categories:
1) Photo
2) Video
3) Audio
4) Behavioral Information
5) Biometric Information
6) Demographic Information

While the first three categories are self-explanatory, the latter three categories are based on user characteristics. We defined behavioral information as observations about the user's activities; biometric information as measurements of the user's body; and demographic information as non-biometric information about the user's traits.

The risks for data types fell into five categories:

1) Financial: the loss of money or property

2) Image: the loss of control over one's self-image (e.g., publicizing something embarrassing)

3) Medical: the disclosure of medical information

4) Physical: physical harm to the user

5) Relationships: damage to inter-personal relationships

After independently coding, researchers met to resolve any disagreements such that the results reflect unanimity. There was $83 \%$ agreement prior to resolution. Cohen's $\kappa$ was 0.81 for the data categories and 0.75 for the risk categories, both indicating "excellent" agreement [18].

With regard to data types, the most concerning type of data is video $(78.0 \%)$, which was ranked similarly to photos $(76.2 \%)$. Next are audio $(66.8 \%)$ and demographic data $(65.4 \%)$, followed by behavioral $(53.1 \%)$ and biometric $(46.3 \%)$ data. We suspect that demographic data was more concerning because it included information such as a Social Security Number, bank account information, and other financial information. We chose to categorize them as such as they are non-biological descriptors of the user. We were surprised that biometric information was seen as benign. We suspect most users today may have an inaccurate understanding of the risks if biometric data is stolen and abused.

\begin{tabular}{|l|r|r|r|}
\hline Parameters & $\chi^{2}$ & $d f$ & QIC \\
\hline \hline (Intercept) & 442.66 & 1 & $12,727.42$ \\
Risk & 405.18 & 4 & \\
\hline (Intercept) & 380.39 & 1 & $12,681.86$ \\
Data Category & 439.45 & 5 & \\
\hline (Intercept) & 256.15 & 1 & $12,061.87$ \\
Risk & 157.84 & 4 & \\
Data Category & 183.90 & 5 & \\
Risk $\times$ Data Category & 259.81 & 8 & \\
\hline (Intercept) & 62.65 & 1 & $10,406.35$ \\
Risk & 205.21 & 4 & \\
Data Category & 250.35 & 5 & \\
Recipient & 546.89 & 3 & \\
IUIPC (covariate) & 103.94 & 1 & \\
Gender (covariate) & 9.80 & 1 & \\
IUIPC $\times$ Gender & 8.21 & 1 & \\
Risk $\times$ Data Category & 303.44 & 8 & \\
Recipient $\times$ Risk & 39.14 & 12 & \\
\hline
\end{tabular}

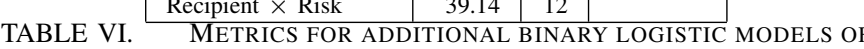
OUR DATA USING GENERAL ESTIMATING EQUATIONS TO ACCOUNT FOR REPEATED MEASURES. THE COLUMNS REPRESENT THE WALD TEST STATISTIC FOR EACH PARAMETER AND THE OVERALL QUASI-AKAIKE INFORMATION CRITERION (QIC) FOR EACH MODEL. EACH PARAMETER LISTED WAS STATISTICALLY SIGNIFICANT AT $p<0.005$.

With regard to the presented risks, we observed that average VURs were highest for financial information disclosure $(82.0 \%)$. Information regarding relationships (69.2\%), physical safety $(66.4 \%)$, and self-image $(65.8 \%)$ followed. VURs were lowest for medical information disclosure $(47.4 \%)$. One reason why medical risks were ranked relatively low is that this category broadly covered scenarios involving data about the user's health, but also included more basic medical information, such as age, gender, and emotional state.

Using these two new variables as additional independent variables (and removing the previous data type variable), we created a second set of models. Because these risk categories and mediums are less likely to change over time, models that take these into account are likely more useful and less likely to be overfit. What these models show us is that both risk and medium are relatively strong predictors by themselves, and have an even stronger interaction effect. When the data recipient and covariates are added to the model, the resulting goodness-of-fit is not much worse than that of the model using the actual data type.

\section{Discussion}

Limitations One of the limitations of our experiment is that our participants might not have knowledge of or interest in wearables and their capabilities; $83 \%$ of our participants reported that they do not own a wearable device. Because of this, our participants may be overestimating or underestimating risk due to an unawareness of what can be inferred from the data collected by these devices. Our participants may not have clear understandings of new technologies with respect to familiar ones, and may also have a higher likelihood of being influenced by reports of recent events relevant to these unfamiliar devices. ${ }^{1}$ We also noted that biometrics were generally not a concern for our participants, although they have security and privacy implications [34]. Our participants also did not differentiate between the benefits and risks of various new capabilities made possible by wearable devices.

\footnotetext{
${ }^{1}$ At the time of the survey, stories of exploding batteries were in the news [26], which were explicitly reported as a concern in our open-ended question.
} 
We recruited both wearable users and non-users to yield a representative sample of the general population. We could have easily recruited only wearables owners or people specifically interested in wearables. However, that would have its own biases and limitations. At the time of this writing, about $85 \%$ of the general population do not own wearable devices [31], [10], indicating our study is reflective of the current population.

Because of the privacy paradox, participants' stated responses may differ from how they may react to these same scenarios in real life [32], [22]. At the same time, our results do reflect actual perceptions of wearable devices and the associated information disclosure scenarios. This is an unavoidable, yet important distinction to make with studies of this nature: our primary goal was to examine perceptions and acceptance so that future systems can be designed with these in mind. We do not expect that such systems will satisfy users in all situations, however, we believe that user-centered design will still be a vast improvement over post hoc approaches (or ignoring user concerns altogether).

Although we presented our participants with a prompt illustrating all the benefits of a wearable device, the questions we asked isolated the risk from the benefits of sharing data with a particular recipient. We sacrificed context due to the complexity of the question necessary for the participant to answer correctly. Users are willing to tolerate risks if there is enough benefit associated with that risk. We do believe that since all of our questions were out of context, our study does represent what data people unambiguously would like to be private and secure, and is accurate for measuring user perceptions of wearables.

Future Research Directions Further work can be done to expand various aspects of this study. A study designed to highlight the differences in information disclosure acceptance, privacy preferences, or technical fluency between wearables owners and non-owners will compliment this work. Investigating more fine-grained data (e.g., investigating specific instances of location data, versus location data in general), additional recipients (e.g., "advertisers" or "acquaintances") may lead to more nuanced results. Additionally, the open-ended concerns illuminate areas of possible future research, such as the design of a distraction-free interface to prevent safety issues, and how to minimize negative social impact.

We find that although people have opinions on applications which are familiar, users are still unfamiliar with particular data types and capabilities. We hope our work both informs the direction for future research to secure video, audio, and other currently considered sensitive sensor input channels, but also encourages work broadly in contextual and user-inputindependent permission models and access control schemes.

\section{RELATED WORK}

User Perceptions While risk communication for the physical world has been examined for several decades (e.g., [39], [16], [28]), research into effectively communicating computerbased risks has only recently been researched. For example, both Garg et al. and Blythe et al. show that due to varying perceptions and abilities that correlate with demographic factors, computer-based risk communication should employ some degree of demographic targeting [19], [5]. While this work is likely applicable to wearable computing risk communication, we believe that a better understanding of users' information risk acceptance in this domain is warranted prior to examining risk communication.

Our study is limited to owners of general consumer wearable devices. Denning et al. study the effect of wearables on bystanders, to find that bystanders have a range of indifferent to negative reactions which depend on how acceptable users find the recording to be [13]. Nasir et al. specifically explore medical wearables, to find that perceived risk determines physician and user acceptance of wearable health technologies [30].

One limitation of user perceptions is that people do not always have enough information to make privacy-sensitive decisions. Even if users did have this information, it has been shown that users often trade off long-term privacy for short-term benefits [3]. Furthermore, actual behavior may deviate from stated privacy preferences [40]. However, understanding user concerns is a necessary first step not only for risk communication, but preventative measures against breaches of privacy and security in a new threat landscape.

Ubiquitous Sensing We are rapidly moving towards a world of ubiquitous sensing and data capture, with ensuing privacy challenges [1], [33], [8]. Roesner et al. urge the community to address potential concerns for wearable devices before the technologies become widespread [36] and explore the unique problems present in terms of law and policy [35]. Privacy preservation research in this age of ubiquitous sensing include frameworks to design for privacy [4], [9], [25], protocols for anonymous communication [11], evaluation metrics for privacy [38], and privacy models [21], [23]. Our work aims to guide these efforts with an insight into user acceptance of common information risks.

Lessons from Smartphones Not long ago, smartphones revolutionized applications' access to data. While this tends to benefit users, they often do not think of the privacy implications. There are still many unresolved concerns such as the opaqueness that prevents users from fully understanding how applications are using their data or rogue applications inappropriately accessing data [24], [42], which are applicable to wearables.

Felt et al. previously studied the security concerns of smartphone users by conducting a large-scale online survey [15]. Their survey asked 3,115 smartphone users about 99 risk scenarios. Participants were asked how upset they would be if a certain action occurred without their permission. Participants rated each situation on a Likert scale ranging from "indifferent (1)" to "very upset (5)." Our methodology closely follows that study, but with scenarios chosen to shed light on the security and privacy risks of wearable devices.

\section{CONCLUSION}

Our survey of 1,784 Internet users is the first largescale study to investigate user-centric information disclosure concerns in the age of wearable computing. We contribute 
a comprehensive ranking of possible risks associated with wearable devices, across various recipients. Our open-ended responses show that privacy and security are at the top of users' overall concerns. While wearables are still in their infancy, perceptions of situations and capabilities are likely to change rapidly with advancements and increased exposure. Our examination of possible data concerns corroborate previous studies of smartphone users that found that video capture and financial data are the most sensitive data types, and least acceptable to be disclosed without user consent. Various systems which detect and take actions for sensitive objects in photos and videos will be critical as wearables and other devices become more ubiquitous. We also found that users' self-reported privacy preferences are correlated with how they may react, even with respect to situations that they are unfamiliar with. Our results may be used by system designers to create permissions and access control mechanisms that do not directly depend on users' inputs. We hope that this work has given an insight into user acceptability in information disclosure and general user concerns about wearable devices, and that this study motivates future privacy and security work for wearable devices.

\section{ACKNOWLEDGEMENTS}

We would like to thank the National Science Foundation (NSF) for supporting the first author through a Graduate Research Fellowship. This work was also made possible by the Intel Science and Technology Center for Secure Computing and NSF awards CNS-1514211 and CNS-1514457.

\section{REFERENCES}

[1] G. D. Abowd and E. D. Mynatt, "Charting past, present, and future research in ubiquitous computing," ACM Transactions on Computer Human Interaction (TOCHI), vol. 7, no. 1, pp. 29-58, 2000.

[2] G. Abramovich, "15 mind-blowing stats about wearable technology," http://www.cmo.com/articles/2014/6/16/Mind_Blowing_Stats_ Wearable_Tech.html, accessed: 2014-12-19.

[3] A. Acquisti and J. Grossklags, "Privacy and rationality in individual decision making," IEEE Security \& Privacy, vol. 2, pp. 24-30, 2005.

[4] V. Bellotti and A. Sellen, "Design for privacy in ubiquitous computing environments," in Proceedings of the Third European Conference on Computer-Supported Cooperative Work 13-17 September 1993, Milan, Italy ECSCW'93. Springer, 1993, pp. 77-92.

[5] J. Blythe, J. Camp, and V. Garg, "Targeted risk communication for computer security," in Proceedings of the 16th International Conference on Intelligent User Interfaces, ser. IUI '11. New York, NY, USA: ACM, 2011, pp. 295-298. [Online]. Available: http://doi.acm.org/10.1145/1943403.1943449

[6] S. Bogaty, "Wearable tech device awareness surpasses 50 percent among us consumers, according to npd," https://www.npd.com/wps/portal/npd/ us/news/press-releases/wearable-tech-device-awareness-surpasses-50percent-among-us-consumers-according-to-npd/, accessed: 2014-12 26.

[7] R. Böhme and J. Grossklags, "The security cost of cheap user interaction," in Proceedings of the 2011 workshop on New security paradigms workshop. ACM, 2011, pp. 67-82.

[8] J. Camp and Y. Chien, "The internet as public space: concepts, issues, and implications in public policy," ACM SIGCAS Computers and Society, vol. 30, no. 3, pp. 13-19, 2000.

[9] L. J. Camp, "Designing for trust," in Trust, Reputation, and Security: Theories and Practice. Springer, 2003, pp. 15-29.

[10] J. Comstock, "Pwc: 1 in 5 americans owns a wearable, 1 in 10 wears them daily," http://mobihealthnews.com/37543/pwc-1-in-5-americansowns-a-wearable-1-in-10-wears-them-daily/, accessed: 2014-12-19.
[11] C. Cornelius, A. Kapadia, D. Kotz, D. Peebles, M. Shin, and N. Triandopoulos, "Anonysense: privacy-aware people-centric sensing," in Proceedings of the 6th international conference on Mobile systems, applications, and services. ACM, 2008, pp. 211-224.

[12] T. Denning, Z. Dehlawi, and T. Kohno, "In situ with bystanders of augmented reality glasses: Perspectives on recording and privacymediating technologies," in Proceedings of the 32nd annual ACM conference on Human factors in computing systems. ACM, 2014, pp. $2377-2386$.

[13] _ _ "In situ with bystanders of augmented reality glasses: Perspectives on recording and privacy-mediating technologies," in Proceedings of the 32Nd Annual ACM Conference on Human Factors in Computing Systems, ser. CHI '14. New York, NY, USA: ACM, 2014, pp. 23772386. [Online]. Available: http://doi.acm.org/10.1145/2556288.2557352

[14] C. Doug Gross, "Google glass targeted as symbol by anti-tech crowd cnn.com," 2014. [Online]. Available: http://www.cnn.com/2014/04/14/ tech/mobile/google-glass-attack/

[15] A. P. Felt, S. Egelman, and D. Wagner, "I've got 99 problems, but vibration ain't one: a survey of smartphone users' concerns," in Proceedings of the second ACM workshop on Security and privacy in smartphones and mobile devices. ACM, 2012, pp. 33-44.

[16] B. Fischhoff, P. Slovic, S. Lichtenstein, S. Read, and B. Combs, "How safe is safe enough? a psychometric study of attitudes towards technological risks and benefits," Policy sciences, vol. 9, no. 2, pp. 127152, 1978.

[17] _ _ "How safe is safe enough? a psychometric study of attitudes towards technological risks and benefits," Policy sciences, vol. 9, no. 2, pp. 127-152, 1978.

[18] J. L. Fleiss, B. Levin, and M. C. Paik, Statistical Methods for Rates and Proportions, 3rd ed. John Wiley \& Sons, Inc., 2003.

[19] V. Garg, L. J. Camp, K. Connelly, and L. Lorenzen-Huber, "Risk communication design: Video vs. text," in Proceedings of the 12th International Conference on Privacy Enhancing Technologies, ser. PETS'12. Berlin, Heidelberg: Springer-Verlag, 2012, pp. 279-298. [Online]. Available: http://dx.doi.org/10.1007/978-3-642-31680-7_15

[20] K. Hill, "Fitbit moves quickly after users' sex stats exposed," http://www.forbes.com/sites/kashmirhill/2011/07/05/fitbit-movesquickly-after-users-sex-stats-exposed/, accessed: 2014-12-26.

[21] J. I. Hong, J. D. Ng, S. Lederer, and J. A. Landay, "Privacy risk models for designing privacy-sensitive ubiquitous computing systems," in Proceedings of the 5th conference on Designing interactive systems: processes, practices, methods, and techniques. ACM, 2004, pp. 91100.

[22] C. Jensen, C. Potts, and C. Jensen, "Privacy practices of internet users: Self-reports versus observed behavior," International Journal of HumanComputer Studies, vol. 63, no. 1, pp. 203-227, 2005.

[23] X. Jiang, J. I. Hong, and J. A. Landay, "Approximate information flows: Socially-based modeling of privacy in ubiquitous computing," in Ubicomp 2002: ubiquitous computing. Springer, 2002, pp. 176-193.

$\begin{array}{llll}\text { [24] S. Kane, "Your apps are watching you," } \\ \text { 2010. } & \text { [Online]. } & \text { Available: } & \text { http://www.wsj.com/articles/ }\end{array}$ SB10001424052748704694004576020083703574602

[25] M. Langheinrich, "Privacy by Design-Principles of Privacy-Aware Ubiquitous Systems," in Ubicomp 2001: Ubiquitous Computing. Springer, 2001, pp. 273-291.

[26] A. Levin, "Exploding lithium batteries riskier to planes: Research," 2014. [Online]. Available: http://www.bloomberg.com/news/articles/ 2014-08-11/exploding-lithium-batteries-riskier-to-planes-research

[27] N. K. Malhotra, S. S. Kim, and J. Agarwal, "Internet users' information privacy concerns (iuipc): the construct, the scale, and a causal model," Information Systems Research, vol. 15, no. 4, pp. 336-355, 2004.

[28] M. G. Morgan, B. Fischhoff, A. Bostrom, and C. Atman, Risk Communication: A Mental Models Approach. New York: Cambridge University Press, 2001.

[29] E. Morphy, "Google glass drops facial recognition (for now)," http://www.forbes.com/sites/erikamorphy/2013/06/02/google-glassdrops-facial-recognition-for-now/, accessed: 2014-12-26.

[30] S. Nasir and Y. Yurder, "Consumers' and physicians' perceptions about high tech wearable health products," Procedia-Social and Behavioral Sciences, vol. 195, pp. 1261-1267, 2015. 
[31] N. News, "Are consumers really interested in wearing tech on their sleeves?" http://www.nielsen.com/us/en/insights/news/2014/tech-stylesare-consumers-really-interested-in-wearing-tech-on-their-sleeves.html, accessed: 2014-12-19.

[32] P. A. Norberg, D. R. Horne, and D. A. Horne, "The privacy paradox: Personal information disclosure intentions versus behaviors," Journal of Consumer Affairs, vol. 41, no. 1, pp. 100-126, 2007.

[33] L. Palen and P. Dourish, "Unpacking Privacy for a Networked World," in Proceedings of the SIGCHI conference on Human factors in computing systems. ACM, 2003, pp. 129-136.

[34] S. Prabhakar, S. Pankanti, and A. K. Jain, "Biometric recognition: Security and privacy concerns," IEEE Security \& Privacy, vol. 1, no. 2, pp. 33-42, 2003.

[35] F. Roesner, T. Kohno, T. Denning, R. Calo, and B. C. Newell, "Augmented reality: hard problems of law and policy," in Proceedings of the 2014 ACM International Joint Conference on Pervasive and Ubiquitous Computing: Adjunct Publication. ACM, 2014, pp. 1283-1288.

[36] F. Roesner, T. Kohno, and D. Molnar, "Security and privacy for augmented reality systems," Communications of the ACM, vol. 57, no. 4, pp. 88-96, 2014.

[37] K. Russell, "I was assaulted for wearing google glass in the wrong part of san francisco," 2014. [Online]. Available: http://www. businessinsider.com/i-was-assaulted-for-wearing-google-glass-2014-4

[38] J. Scholtz and S. Consolvo, "Toward a framework for evaluating ubiquitous computing applications," Pervasive Computing, IEEE, vol. 3, no. 2, pp. 82-88, 2004.

[39] P. E. Slovic, The perception of risk. Earthscan publications, 2000.

[40] S. Spiekermann, J. Grossklags, and B. Berendt, "E-privacy in 2nd generation e-commerce: Privacy preferences versus actual behavior," in Proceedings of the 3rd ACM conference on Electronic Commerce. ACM, 2001, pp. 38-47.

[41] H. R. Varian, F. Wallenberg, and G. Woroch, "The demographics of the do-not-call list." IEEE Security \& Privacy, vol. 3, no. 1, pp. 34-39, 2005.

[42] Y. Zhou, X. Zhang, X. Jiang, and V. W. Freeh, "Taming informationstealing smartphone applications (on android)," in Trust and Trustworthy Computing. Springer, 2011, pp. 93-107.

\section{APPENDIX}

Omitted Survey Questions Our participants answered four additional questions not detailed in this paper. Two questions compared smartphones to wearable devices, to investigate if participants inherently saw wearables as threatening due to its unfamiliarity. The questions were not particularly well suited for this task, and our results showed no significant difference between smartphone and wearables perceptions.

The other two questions asked participants to numerically rate risks and benefits of familiar technologies versus wearables (e.g., an airplane versus a wearable). This was to mimic the methodology in Fischhoff's seminal study on attitudes toward technological risks and benefits. In Fischhoff's study [17], all technologies were concerning with physical risk, while ours mixed information disclosure risks with physical risks. These comparisons were unsound to make. Nonetheless, our results largely highlighted participants' unfamiliarity with devices; generally, participants rated more familiar technologies as more beneficial or risky.

Coding Label Definitions To give some transparency into how we coded the answers (ranging from 1 to 1,400+ words), we provide the coding outline we used. An answer mentioning multiple concerns was given multiple coding labels.
Privacy: "privacy," mention of personal details, spying.

Security: "security," mention of malware, hacking.

GPS tracking: "location," "GPS," mention of monitoring.

Being Unaware: mention of using, collecting, and disclosing data without permission.

False information: inaccurate or maliciously false data.

Health Risk: mention of radiation, cancer, or other effects.

Safety: mention of distractions causing car crashes and injuries, violence due to the device, injuries from malfunctions.

Discomfort: mention of eye strain, headache, irritation.

Financial cost: cost of buying or using the device.

Theft: mention of device theft.

Social Impact: mention of dependency, distance from people, changes in decision making, etc.

Social Stigma: mention of judgment, hate, or bystanders.

Aesthetics: mention of fashion or looking dorky.

Miscellaneous: odd comments, uncommon concerns.

None: "None," mention of no threat, or no real concerns

Don't know: "do not know," general confusion

Don't care: " do not care," nonchalant answers 


\begin{tabular}{|c|c|c|c|c|c|}
\hline Question & All & Friends & Public & Work & App \\
\hline video of you unclothed & $95 \%(1)$ & $97 \%(4)$ & $94 \%(10)$ & $100 \%(1)$ & $90 \%(2)$ \\
\hline bank account information & $95 \%(2)$ & $94 \%(10)$ & $95 \%(7)$ & $100 \%(1)$ & $90 \%(1)$ \\
\hline social security number & $94 \%(3)$ & $100 \%(1)$ & $100 \%(1)$ & $93 \%(9)$ & $88 \%(3)$ \\
\hline video entering in a PIN at an ATM & $92 \%(4)$ & $100 \%(1)$ & $93 \%(12)$ & $87 \%(20)$ & $88 \%(4)$ \\
\hline photo of you unclothed & $92 \%(5)$ & $96 \%(6)$ & $91 \%(16)$ & $100 \%(1)$ & $77 \%(6)$ \\
\hline photo of you that is very embarrassing & $91 \%(6)$ & $94 \%(8)$ & $100 \%(1)$ & $94 \%(6)$ & $78 \%(5)$ \\
\hline username and password for websites & $89 \%(7)$ & $96 \%(5)$ & $95 \%(9)$ & $94 \%(7)$ & $64 \%(14)$ \\
\hline credit card information & $88 \%(8)$ & $100 \%(1)$ & $93 \%(13)$ & $95 \%(5)$ & $65 \%(13)$ \\
\hline video of you that is very embarrassing & $88 \%(9)$ & $91 \%(13)$ & $94 \%(11)$ & $94 \%(7)$ & $71 \%(9)$ \\
\hline photo of you at home & $87 \%(10)$ & $85 \%(19)$ & $96 \%(5)$ & $93 \%(10)$ & $71 \%(10)$ \\
\hline audio recording of work conversations & $86 \%(11)$ & $94 \%(9)$ & $96 \%(6)$ & $100 \%(1)$ & $53 \%(24)$ \\
\hline video of entering in a passcode to a door & $85 \%(12)$ & $95 \%(7)$ & $89 \%(21)$ & $81 \%(35)$ & $75 \%(7)$ \\
\hline audio recording of phone conversations & $85 \%(13)$ & $93 \%(11)$ & $97 \%(4)$ & $90 \%(14)$ & $56 \%(20)$ \\
\hline amount of money you have & $84 \%(14)$ & $90 \%(14)$ & $100 \%(1)$ & $93 \%(11)$ & $63 \%(15)$ \\
\hline video of you intoxicated & $83 \%(15)$ & $81 \%(26)$ & $91 \%(16)$ & $88 \%(17)$ & $68 \%(11)$ \\
\hline when you have sex & $81 \%(16)$ & $78 \%(31)$ & $87 \%(23)$ & $90 \%(15)$ & $73 \%(8)$ \\
\hline how much debt you have & $81 \%(17)$ & $85 \%(19)$ & $90 \%(20)$ & $87 \%(22)$ & $59 \%(18)$ \\
\hline video of you at home & $81 \%(18)$ & $87 \%(16)$ & $86 \%(24)$ & $89 \%(16)$ & $60 \%(17)$ \\
\hline photo of you intoxicated & $78 \%(19)$ & $80 \%(27)$ & $90 \%(18)$ & $87 \%(23)$ & $53 \%(25)$ \\
\hline photo of you at random & $78 \%(20)$ & $82 \%(24)$ & $83 \%(29)$ & $81 \%(32)$ & $66 \%(12)$ \\
\hline audio recording of conversations & $78 \%(21)$ & $86 \%(18)$ & $85 \%(26)$ & $87 \%(20)$ & $55 \%(21)$ \\
\hline medical conditions & $77 \%(22)$ & $92 \%(12)$ & $85 \%(25)$ & $85 \%(27)$ & $40 \%(37)$ \\
\hline video of you at random & $76 \%(23)$ & $73 \%(40)$ & $90 \%(19)$ & $88 \%(19)$ & $48 \%(31)$ \\
\hline video of you off-guard & $76 \%(24)$ & $85 \%(21)$ & $79 \%(34)$ & $91 \%(13)$ & $53 \%(23)$ \\
\hline photo of your work or workplace & $74 \%(25)$ & $76 \%(33)$ & $82 \%(31)$ & $81 \%(32)$ & $62 \%(16)$ \\
\hline username for websites & $73 \%(26)$ & $90 \%(15)$ & $74 \%(43)$ & $84 \%(28)$ & $50 \%(29)$ \\
\hline address & $72 \%(27)$ & $62 \%(50)$ & $93 \%(14)$ & $81 \%(31)$ & $51 \%(28)$ \\
\hline audio recording you captured & $72 \%(28)$ & $87 \%(17)$ & $75 \%(40)$ & $72 \%(46)$ & $50 \%(29)$ \\
\hline photo of you off-guard & $72 \%(29)$ & $83 \%(23)$ & $80 \%(32)$ & $80 \%(37)$ & $45 \%(33)$ \\
\hline photo downloaded from internet & $71 \%(30)$ & $79 \%(29)$ & $76 \%(38)$ & $86 \%(25)$ & $32 \%(47)$ \\
\hline photo others sent you & $71 \%(31)$ & $85 \%(21)$ & $84 \%(27)$ & $75 \%(44)$ & $41 \%(35)$ \\
\hline video others sent you & $70 \%(32)$ & $82 \%(24)$ & $95 \%(7)$ & $80 \%(37)$ & $30 \%(49)$ \\
\hline video of your work or workplace & $70 \%(33)$ & $74 \%(36)$ & $83 \%(28)$ & $70 \%(49)$ & $51 \%(26)$ \\
\hline fingerprint & $70 \%(34)$ & $77 \%(32)$ & $80 \%(32)$ & $70 \%(48)$ & $55 \%(22)$ \\
\hline when you were lying nervous or stressed & $69 \%(35)$ & $74 \%(35)$ & $74 \%(42)$ & $91 \%(12)$ & $41 \%(34)$ \\
\hline audio recording of you $\%$ (voice notes) & $69 \%(36)$ & $80 \%(28)$ & $78 \%(35)$ & $88 \%(18)$ & $38 \%(39)$ \\
\hline medication taken & $69 \%(37)$ & $79 \%(29)$ & $73 \%(44)$ & $81 \%(34)$ & $37 \%(40)$ \\
\hline videos taken on device & $68 \%(38)$ & $58 \%(52)$ & $82 \%(30)$ & $79 \%(40)$ & $51 \%(27)$ \\
\hline photo of your signature & $68 \%(39)$ & $63 \%(48)$ & $64 \%(51)$ & $85 \%(26)$ & $59 \%(19)$ \\
\hline web history & $66 \%(40)$ & $74 \%(36)$ & $70 \%(45)$ & $86 \%(24)$ & $37 \%(40)$ \\
\hline photos already on device & $66 \%(41)$ & $75 \%(34)$ & $77 \%(36)$ & $79 \%(39)$ & $27 \%(53)$ \\
\hline home address & $65 \%(42)$ & $61 \%(51)$ & $87 \%(22)$ & $69 \%(50)$ & $40 \%(36)$ \\
\hline fine-grained location tracking $(+/-\mathrm{cm})$ & $63 \%(43)$ & $73 \%(39)$ & $76 \%(37)$ & $78 \%(41)$ & $30 \%(50)$ \\
\hline photo of people at random & $61 \%(44)$ & $72 \%(41)$ & $61 \%(54)$ & $82 \%(30)$ & $38 \%(38)$ \\
\hline video downloaded from the internet & $61 \%(45)$ & $63 \%(47)$ & $75 \%(40)$ & $82 \%(29)$ & $33 \%(45)$ \\
\hline when you are alone & $61 \%(46)$ & $51 \%(55)$ & $69 \%(46)$ & $80 \%(36)$ & $35 \%(43)$ \\
\hline location tracking $(+/-\mathrm{m})$ & $61 \%(47)$ & $57 \%(53)$ & $92 \%(15)$ & $63 \%(55)$ & $25 \%(56)$ \\
\hline videos of people at random & $61 \%(48)$ & $63 \%(49)$ & $75 \%(39)$ & $71 \%(47)$ & $28 \%(52)$ \\
\hline where you are currently going & $60 \%(49)$ & $74 \%(36)$ & $68 \%(48)$ & $65 \%(54)$ & $35 \%(44)$ \\
\hline recording of sound around you & $60 \%(50)$ & $71 \%(42)$ & $64 \%(50)$ & $75 \%(43)$ & $35 \%(42)$ \\
\hline people you spend time with & $60 \%(51)$ & $71 \%(42)$ & $60 \%(55)$ & $76 \%(42)$ & $31 \%(48)$ \\
\hline workplace address & $58 \%(52)$ & $69 \%(45)$ & $64 \%(49)$ & $57 \%(61)$ & $46 \%(32)$ \\
\hline sounds on device $\%$ (notifications, etc) & $54 \%(53)$ & $70 \%(44)$ & $59 \%(56)$ & $66 \%(52)$ & $22 \%(58)$ \\
\hline phone usage & $51 \%(54)$ & $67 \%(46)$ & $56 \%(57)$ & $68 \%(51)$ & $15 \%(64)$ \\
\hline purchased products & $50 \%(55)$ & $57 \%(54)$ & $55 \%(58)$ & $62 \%(57)$ & $26 \%(54)$ \\
\hline when you are sick or healthy & $48 \%(56)$ & $40 \%(64)$ & $61 \%(52)$ & $62 \%(58)$ & $26 \%(55)$ \\
\hline how close you are to interacting people & $46 \%(57)$ & $50 \%(57)$ & $61 \%(53)$ & $51 \%(62)$ & $13 \%(66)$ \\
\hline feelings (based on biometrics) & $46 \%(58)$ & $50 \%(57)$ & $55 \%(58)$ & $63 \%(56)$ & $18 \%(61)$ \\
\hline computer usage & $44 \%(59)$ & $51 \%(56)$ & $52 \%(60)$ & $45 \%(63)$ & $28 \%(51)$ \\
\hline eating patterns & $42 \%(60)$ & $41 \%(62)$ & $45 \%(62)$ & $75 \%(45)$ & $12 \%(67)$ \\
\hline name & $42 \%(61)$ & $50 \%(57)$ & $68 \%(47)$ & $26 \%(71)$ & $32 \%(46)$ \\
\hline sleeping patterns & $40 \%(62)$ & $43 \%(61)$ & $41 \%(63)$ & $62 \%(59)$ & $21 \%(59)$ \\
\hline eye patterns $\%$ (for eye tracking) & $40 \%(63)$ & $48 \%(60)$ & $50 \%(61)$ & $61 \%(60)$ & $6 \%(71)$ \\
\hline exercise patterns & $38 \%(64)$ & $33 \%(67)$ & $34 \%(66)$ & $66 \%(52)$ & $16 \%(63)$ \\
\hline when you are happy or having fun & $34 \%(65)$ & $40 \%(64)$ & $32 \%(69)$ & $43 \%(65)$ & $24 \%(57)$ \\
\hline television shows watched & $30 \%(66)$ & $38 \%(66)$ & $33 \%(67)$ & $36 \%(68)$ & $11 \%(68)$ \\
\hline when you are busy or interruptible & $29 \%(67)$ & $40 \%(63)$ & $28 \%(70)$ & $36 \%(68)$ & $17 \%(62)$ \\
\hline music on device & $28 \%(68)$ & $4 \%(72)$ & $37 \%(64)$ & $42 \%(66)$ & $20 \%(60)$ \\
\hline heart rate & $27 \%(69)$ & $21 \%(68)$ & $36 \%(65)$ & $44 \%(64)$ & $9 \%(70)$ \\
\hline age & $24 \%(70)$ & $17 \%(69)$ & $33 \%(67)$ & $36 \%(67)$ & $14 \%(65)$ \\
\hline language spoken & $15 \%(71)$ & $17 \%(70)$ & $18 \%(72)$ & $28 \%(70)$ & $27 \%(53)$ \\
\hline gender & $15 \%(72)$ & $15 \%(71)$ & $19 \%(71)$ & $15 \%(72)$ & $9 \%(69)$ \\
\hline
\end{tabular}

